\title{
The Role of Bacterial Biofilms in Infections and Effective Prevention Strategies for Biofilm Formation in Urinary Catheters
}

\section{K. Vivehananthan*, S. Thevashayinath, I. Abeygunawardena}

Department of Basic Sciences, Faculty of Health Sciences, The Open University of Sri Lanka, Nawala, Nugegoda

\section{Abstract}

Biofilm formation is a complicated microbiological process and one of the distinctive features of pathogenic bacteria. Biofilms are a cluster of bacterial cells enclosed in extra polymeric substances and irreversibly attached onto a surface. Biofilms have a major impact on public health as biofilm associated bacteria are able to survive and populate in indwelling medical devices, causing severe nosocomial and recurrent infections. Biofilm-embedded bacteria have unique characteristics which are harder to destroy than the planktonic forms. Especially biofilm bacteria are highly resistant to antimicrobial agents. Treating patients undergoing long term urinary catheterization is becoming complicated by encrustation and blockage due to crystalline biofilm formation inside the lumen of catheters. Further, all types of indwelling catheters including silver or nitrofurazone-coated devices are

${ }^{*}$ Corresponding should be addressed to Dr. K. Vivehananthan, Dept. of Basic Sciences, Faculty of Health Sciences, The Open University of Sri Lanka, Sri Lanka.

Email:kvive@ou.ac.lk

Dhttps://orcid.org/0000-0003-4939-3258

(Received 30th October 2019; Revised 30th April 2020; Accepted 15th May 2020) (C) OUSL) 
vulnerable to biofilm formation.

Therefore, there is an urgent requirement to develop either urinary catheters with other alternative coatings or new urinary drainage systems which may lead to reduction of infections and morbidity. Other than that, controlling biofilm formation is a necessary requirement during both manufacturing and usage of catheters. Antifouling and biocidal coatings on the catheters are currently being investigated and it is proved that herbal products also express anti-biofilm activity.

This review is largely based on previous literature describing biofilm formation and their role in catheter associated urinary tract infection. Further, mechanism of biofilm formation in indwelling urinary catheters, antibiotic resistance patterns, and the detection methods of biofilm are briefly described. Understanding the mechanisms of biofilm formation and the antibiotic resistance patterns is of utmost importance which will result in the development of new remedies for biofilm infection.

Keywords: Antibiotic Resistance, Biofilm Formation, Urinary Catheters

\section{Introduction}

\section{The Structure and Formation of Microbial Biofilms}

Biofilm is defined as accumulation of the microbial organisms that is permanently attached to biotic and abiotic surface and generally enclosed in self-produced extracellular polysaccharide substances (EPS) (Sayal et al., 2014; Wijesinghe et al., 2019). Biofilm production is a complicated microbiological process which is a major pathogenic event in the infection. It involves a sequence of steps, including irreversible attachment, accumulation, and maturation (Alves et al., 2014; Tiwari \& Ghnawate, 2017). When the bacterial colonies attach to surfaces, the bacterial cells upregulate genes which are specific for adhesion. Further, bacteria itself secrete a sticky gum like material for anchorage called as extracellular substances (Sayal et al., 2014; Tiwari \& 
Ghnawate, 2017; Wijesinghe et al., 2019). Also, the bacteria populate by multiplying rapidly and spreading along the surface within the EPS of the biofilm. Some of the bacteria detach from the surface and again become into planktonic form and then attach to a new colonised surface (Tiwari \& Ghnawate, 2017). This phenomenon helps the metastasis process of biofilm.

Initially the thickness of biofilm is approximately $200 \mu \mathrm{m}$ and over time it reaches a thickness of $\sim 500 \mu \mathrm{m}$ (Singha et al., 2017). Types of EPS and the diversity of biofilm vary according to the bacterial species and strain (Osungunna \& Onawunmi, 2018). Staphylococcal polysaccharide, Pseudomonas aeruginosa alginate, streptococcal and lactobacilli glucans and fructans are well known examples for biofilm-associated EPS. Biofilm is composed of small percentage of bacteria compare to EPS which occupies a large portion of biofilm (Tiwari \& Ghnawate, 2017). EPS binds the bacteria together in a sticky web of twisted EPS fibers. The fibers interconnect the cells and anchor them to a surface, therefore EPS is considered as the backbone of biofilm (Murugan et al., 2016). The formation of EPS permits the emerging biofilm community to develop a three-dimensional structure (Murugan et al., 2016; Singha et al., 2017). EPS and the accompanying weblike structure are known as extra cellular matrix or biofilm matrix. Bacterial colonies are found within the biofilm matrix (Tiwari \& Ghnawate, 2017). Physical and the physiological properties of biofilm are determined by the EPS and the bacterial cells respectively (Murugan et al., 2016).

Biofilms have water channels which transport the essential nutrients and oxygen to internal cells (Alves et al., 2014; Tiwari \& Ghnawate, 2017), and remove the waste products and toxins from the cells. Extracellular matrix facilitates communications among the microbial cells via biochemical signals (Tiwari \& Ghnawate, 2017), such as acyl-homoserine lactone and oligopeptides facilitates communication in gram negative bacteria and gram positive bacteria respectively in an event called as quorum sensing (Maharjan et al., 2018). Also, environmental factors have a major impact on formation of biofilm including EPS production 
(Murugan et al., 2016; Singha et al., 2017). The mechanisms of how environmental factors influence biofilm formation yet to be justified (Murugan et al., 2016).

\section{Pathogenesis and Antibiotic Resistance Mechanisms of Biofilms}

Biofilm develop on the surfaces of living tissues such as the urinary bladder and abiotic surfaces generally in indwelling devices (Bardoloi \& Yogeesha Babu, 2017). Biofilms are considered as one of the serious medical threats to patient's health as the matrix prevent the pathogenic microorganism against the host defense mechanism ( Sayal et al., 2014 ; Maharjan et al., 2018) and its unique characteristics enable intrinsic resistance to antibacterial drugs. Biofilm associated bacteria acquire 100- to 1000-fold increase in antibiotic tolerance compared to planktonic bacteria (Murugan et al., 2016). Bacteria become more resistant after adhering to the surface and subsequently develop more resistance over the following days of biofilm production (Tiwari \& Ghnawate, 2017).

Generally biofilm consists of multi drug-resistant bacterial species separated by interstitial fluid (Alves et al., 2014; Bardoloi \& Yogeesha Babu, 2017). Biofilm also consists of gram positive bacteria such as Staphylococcus aureus, Staphylococcus epidermidis, Enterococcus faecalis and Gram negative bacteria such as Escherichia coli, Klebsiella pneumoniae, Proteus mirabilis and Pseudomonas aeruginosa ( Patel et al., 2016 ; Bardoloi \& Yogeesha Babu, 2017).

Biofilm bacteria exhibit different antibiotic resistant strategies than the normal pathogenic bacteria, and the resistance techniques depend on the slow penetration of the antibiotics, different rates of bacterial growth and metabolism as well as horizontal transmission of drug-resistant gene between the closely attached cells (Alves et al., 2014; Tiwari \& Ghnawate, 2017 ; Maharjan et al., 2018). Also, biofilm bacteria have the ability to form unique subpopulation like spore formation. The subpopulations have different phenotypes and persist in a dormant state which are highly resistance to antibiotics and cause recurrent infections (Murugan et al., 2016; Singha et al., 
2017). In addition to that, the biofilm matrix consists of concentrated extracellular enzymes including formaldehyde dehydrogenase, formaldehyde lyase and $\beta$-lactamases. The enzymes are capable of inactivating the hydrophilic positively charged antibacterial substances (Murugan et al., 2016).

A knowledge of biofilm formation and antibiotic resistance profile of bacterial species are critical to find new techniques for therapeutic use. Since the biofilm bacteria is highly resistance to antibiotic, there are considerable drawbacks in the treatments of patients with this type of infection (Osungunna \& Onawunmi, 2018; Sayal et al., 2014). Especially, antibiotic resistivity against commonly used antibiotics for the urinary tract infection has been increasing worldwide. It was found that the resistance pattern may vary with the bacterial stain (Murugan et al., 2011). According to the reports from different countries, drugs Colistin, Ofloxacin, Fosfomycin, Vancomycin, Meropenem, Imipenem, Piperacillin and Tazobactum remain as the antibiotic of choice in the treatment of biofilm infections (Dumaru et al., 2019; Maharjan et al., 2018; Osungunna \& Onawunmi, 2018; Sabir et al., 2017). On account of antibiotic resistance pattern, the highest resistance was observed with Ampicillin and Ceftazidime, followed by Amoxicillin Clavulanate and Tetracycline (Maharjan et al., 2018; Sabir et al., 2017).

\section{Biofilm in Indwelling Urinary Catheters}

Indwelling medical devices support to increase therapeutic results of patients, particularly those requiring life supporting devices and protect their lives, however these devices are more prone to get colonization of biofilm bacteria ( Dror et al., 2009 ; Alves et al., 2014 ; Bardoloi \& Yogeesha Babu, 2017). About 30\% of biofilm producing bacteria have been identified from indwelling medical equipment including endotracheal tubes, central venous catheters and urinary catheters. Among the medical devices, indwelling urinary catheters are known to be the major common site of biofilm producing bacteria ( Patel et al., 2016 ; Bardoloi \& Yogeesha Babu, 2017). The bacteria can originate in the inner or 
outer part of the device (Sayal et al., 2014). The time needed for the biofilm to be produced on the device is based on the bacterial group and the type of device material. But a day is enough to produce a thick biofilm on the complete surface of a polymeric device (Alves et al., 2014; Maharjan et al., 2018).

Urinary tract infections play a major part in hospitalized infections and nearly $97 \%$ of the cases were related to urinary catheters. About $60 \%$ of infections developed due to the presence of biofilm (Alves et al., 2014). Generally during initial admissions at hospitals, around 12 to $16 \%$ of adult inpatients have an indwelling urinary catheter (Maharjan et al., 2018). Almost $50 \%$ of catheterized patients acquire infections within a period of less than one week. Patients catheterized for nearly 4 weeks have a $100 \%$ possibility of getting infections (Alves et al., 2014). A study carried out in India indicates that E.coli (29.74\%) was the most commonly isolated followed by $K$. pneumoniae $(21.84 \%), P$. mirabilis $(15.42 \%)$ from the urine samples of patients with indwelling urinary catheters (Sayal et al., 2014).

If the urinary catheter is inserted in the same area for a longer period, it increases the likelihood of these microorganisms producing biofilms and results in chronic and recurrent urinary tract infections (Sayal et al., 2014). If the infection is not treated, it may consequently lead to bacteraemia, bacterial vaginosis, chronic renal infection, acute pyelonephritis, bladder cancer and in certain cases death (Alves et al., 2014).

\section{Crystalline Biofilm Formation in Urinary Catheters}

Bacteria use the catheter as a pathway to enter into the bladder (Tiwari \& Ghnawate, 2017). Urease is an enzyme produced by bacteria which are colonized in the urine of catheterized patients (Stickler, 2014). Pseudomonas aeruginosa, Klebsiella pneumoniae, Morganella morganii, Proteus species, some Providencia species, Staphylococcus aureus and coagulase-negative Staphylococci produce urease in urine and form a unique type of crystalline bio film on catheters (Stickler, 2014; Tiwari \& Ghnawate, 2017). Especially, Proteus mirabilis is the most common bacteria which forms crystalline biofilms in the urinary catheters (Stickler, 2014). 
Formation of Crystalline biofilms involves in sequences of steps. Initially urease enzyme produced by bacterial species break down the urea in urine and release ammonia. Ammonia increases the $\mathrm{pH}$ level of urine and subsequently results in the formation of calcium and magnesium phosphate crystalline. Accumulation of the crystalline materials occurs in the urine that remains inside the catheter lumen and develop rapidly within the biofilm matrix (Broomfield et al., 2009; Stickler, 2014; Tiwari \& Ghnawate, 2017). Also the crystalline materials harden and obstruct the lumen of catheter and finally block the urine flow. Blockage of urine flow results in painful bladder distension and if the urine from the distended bladder refluxes into the kidney, it causes pyelonephritis (Stickler, 2014; Tiwari \& Ghnawate, 2017). However, if the particular causative agent is not identified it can make the disease more severe and in some cases, it can be fatal (Alves et al., 2014). All available catheter types are vulnerable to encrustation and blockage including silver or nitrofurazonecoated ones (Stickler, 2014). High morbidity and the mortality rates of catheter associated urinary tract infection increase the hospital stay and cost of the treatment (Maharjan et al., 2018).

\section{Detection Methods of Biofilm}

Conventional methods are economical and reliable for the routine screening and diagnosis (Dumaru et al., 2019). Biofilm can be easily demonstrated by phenotypic methods such as tube method, Congo Red Agar (CRA) method and tissue culture plate method. Besides that genotypic methods have also been carried out to screen biofilm production as indirect proof of the presence of biofilms (Bardoloi \& Yogeesha Babu, 2017). Molecular methods considerably widened a spectrum of microbes detected in biofilms. The Modern Next-Generation Sequencing (NGS) techniques is beneficial in studying highly diverse bacterial communities (Kotaskova et al., 2019). NGS targeting 16S rDNA is able to analyse the bacterial composition in catheter related infection and therefore it is suggested as a promising diagnostic tool for catheter related infections (Okuda et al., 2018). 
The new test methods are currently used for biofilm detection such as DNA based methods including quantitative real time PCR, multiplex PCR, Sanger sequencing, next generation sequencing and micro array analysis. Further, confocal scanning, laser microscopy with molecular probes, Fluorescence In Situ Hybridization (FISH) or labelled antibodies-electron microscopy are used to detect biofilms (Magana et al., 2018; Petrachi et al., 2017). Virulence genes responsible for biofilm formation can be amplified with the usage of gene specific primers, even in the uncultured pathogen present in the sample (Kırmusaoğlu, 2019). Researches who worked with biofilm structures have identified virulence genes responsible for biofilm formation such as cup A gene in $P$. aeruginosa, ica gene in $S$. aureus and esp gene in $E$. faecalis (Falaki \& Mahdavi, 2017; Murugan et al., 2016; PérezOsorio et al., 2010). 16S rDNA sequencing is the most common housekeeping genetic marker to identify bacterial species. 16S rDNA molecule among various bacteria have highly conserved nucleotide sequence interspersed with regions of more variables that are specific to genes or species (Jenkins et al., 2012; Kai et al., 2018; Kotaskova et al., 2019; Maciel et al., 2009). Each bacterial species has its unique 16S rDNA sequences with differences (<0.5\%) (Janda \& Abbott, 2007). Phylogenetic analysis using 16S rDNA sequences is considered to be a highly reliable and important bioinformatics tool (Roy et al., 2014; Yang et al., 2016).

Phylogenetic is the study of the evolutionary relatedness among groups of organisms. Molecular phylogenetic uses sequence data to infer these relationships for both organisms and the genes they maintain (Ziemert \& Jensen, 2012). Evolutionary relationship among the biofilm bacterial species are determined by phylogenetic analysis (Murugan et al., 2016). Phylogenetic tree is a branching diagram where the simple tree representation of the evolution makes the phylogenetic analysis easier to comprehend and represent as well (Roy et al., 2014).

\section{Effective Strategies and Novel Approaches to Prevent Biofilm Formation}

Nowadays mortality rates increase and there is an urgent 
requirement to develop strategies to prevent and eradicate biofilm production on urinary catheters (Alves et al., 2014). In this standpoint, early detection of biofilm producers is crucial (Maharjan et al., 2018). Also analysing the biofilm development with regard to age, gender, hospital unit, bacterial species (Alves et al., 2014) and their antibiotic susceptibility may provide a wide knowledge regarding treatment and prevention of such infections (Sayal et al., 2014). Several studies indicate that microbial studies alone will not eradicate CAUTIs (Singha et al., 2017). In order to meet all the challenges of catheter associated infection, development of an alternative indwelling catheter system is an important requirement which should have the same mechanism as natural physiology of urine elimination and emptying urine completely without damaging the bladder and appropriate for all users (Feneley et al., 2015). Researchers need to understand the interaction between the pathogens and materials with regard to development of infection. The necessity for better catheter materials has significantly increased (Singha et al., 2017).

A research study reports that Azithromycin-Ciprofloxacinimpregnated urinary catheters inhibit bacterial colonization, biofilm formation, and inflammation in a murine model of foreign-bodyassociated urinary tract infections caused by Pseudomonas aeruginosa (Saini et al., 2017). Another research study indicates that silverpolytetrafluoroethylene (Ag-PTFE) coated catheters substantially inhibit encrustation compare to uncoated ones. Currently coated catheters which are effective for long term catheterization are commercially not available. Silver has antibacterial properties (Singha et al., 2017). Silver coated catheters are available in the market, but antibacterial properties of them are inactivated due to the sticky biofilm formation on the extra luminal part of the catheters. PTFE has an excellent non-stick properties and hence it is suggested that the Ag-PTFE would be a promising antibacterial coating for urinary catheters (Wang et al., 2019). Research on Antifouling and biocidal materials would be most useful in designing competent and simple catheters. Antifouling coatings have the ability to prevent bacterial attachment to the surfaces, 
whereas biocidal materials kill the pathogens. Silver ions, Triclosan, Chlorhexidine, Chlorine, Tributyltin and Nitric oxide are the chemicals which show biocide activity (Singha et al., 2017). It has been proven in a research study that Chlorhexidine sustained-release varnish-coated urinary catheters efficaciously reduce urinary catheter-associated biofilm formation (Segev et al., 2013).

Besides that, discovering natural agents could provide novel strategies for catheter associated biofilm infections. Research reports from various countries indicate that significant amount of plant species have anti-bacterial and anti-biofilm activity. Garlic shows inhibitory effect in quorum sensing mechanism which plays a vital role in biofilm formation in bacteria. Moreover, plant Cocculas trilobus has the ability of blocking the adherence of bacteria onto surfaces. As well as Cranberry Polyphenols, Herba parriniae extract, and Ginkgo biloba extract have strong inhibitory effects on biofilm formation (Lu et al., 2019). Furthermore it is proved that catheters impregnated with herbal plant powders including Andrographis paniculata, Euphorbia hirta and Terminalia chebula have antibiofilm activity. Seeking novel antimicrobials from ethnomedicinal plant may provide a new pathway for preventing biofilm infections (AnilKumar, 2015).

\section{Conclusions}

Almost all cases of CAUTI are biofilm-related infections (Bardoloi \& Yogeesha Babu, 2017). Biofilms and their control procedures have been mentioned in several aforementioned studies and complications of biofilm are thought provoking and which raises serious concerns (Maharjan et al., 2018). Understanding the nature of biofilm and their connection with the environmental factors may help to discover treatment and management methods for CAUTI (Murugan et al., 2016). In Sri Lanka, even though few works have been reported on utilizing the beneficial effect of biofilm bacteria (eg: biofilm biofertilizers) in the field of agriculture (Herath et al, 2013), there are lack of research done on biofilm infection in health-related field. 
Diagnosis and the prevention methods of catheter associated urinary tract infection require expertise, facilities and surveillance activities. Due to inadequate surveillance and reporting system, magnitude of catheter associated UTI has been underestimated and developed into a common problem in the developing countries. Lack of knowledge with regard to development of microorganisms in biofilms lead to slow progression of detecting biofilms in medical devices (Awoke et al., 2019). Therefore, research related to catheter associated biofilm is most important and it will raise the awareness of this condition to the general public of Sri Lanka.

\section{References}

Alves, M. J., Barreira, J. C. M., Carvalho, I., Trinta, L., Perreira, L., Ferreira, I. C. F. R. \& Pintado, M. (2014). Propensity for biofilm formation by clinical isolates from urinary tract infections: Developing a multifactorial predictive model to improve antibiotherapy. Journal of Medical Microbiology, 63 (3), 471-477. https://doi.org/10.1099/jmm.0.0 71746-0

AnilKumar, S., Getharamani, \& Elayarajah, B. (2015). Fabrication of Antibacterial Coatings: Prevention of Implant Associated Infections in Patients Indwelling Urinary Catheters. IOSR Journal of Pharmacy and Biological Sciences (IOSR-JPBS), 10 (6), 82-89. https://doi.org/10.9790/30 08-10638289

Awoke, N., Kassa, T. \& Teshager, L. (2019). Magnitude of Biofilm Formation and Antimicrobial Resistance Pattern of Bacteria Isolated from Urinary Catheterized Inpatients of Jimma University Medical Center, Southwest Ethiopia. International Journal of Microbiology, 2019. https:// doi.org/10.1155/2019/5729568

Bardoloi, V. \& Yogeesha Babu, K. V. (2017). Comparative study of isolates from community-acquired and catheter-associated urinary tract infections with reference to biofilm-producing 
property, antibiotic sensitivity and multi-drug resistance. Journal of Medical Microbiology, 66 (7), 927-936. https:// doi.org/10.1099/jmm.0.000525

Broomfield, R. J., Morgan, S. D., Khan, A. \& Stickler, D. J. (2009). Crystalline bacterial biofilm formation on urinary catheters by urease-producing urinary tract pathogens: A simple method of control. Journal of Medical Microbiology, 58, 1367-1375. https://doi.org/10.1099/jmm.0.0 12419-0

Dror, N., Mandel, M., Hazan, Z. \& Lavie, G. (2009). Advances in Microbial Biofilm Prevention on Indwelling Medical Devices with Emphasis on Usage of Acoustic Energy. Sensors, 9, 2538-2554. https://doi.org/10.3390/s 90402538

Dumaru, R., Baral, R. \& Shrestha, L. B. (2019). Study of biofilm formation and antibiotic resistance pattern of gramnegative Bacilli among the clinical isolates at BPKIHS, Dharan. BMC Research Notes, 12 (1), 1-6. htt ps:// doi. org/10.1186/s13104-019-4084-8

Falaki, B. \& Mahdavi, S. (2017). Study of Distribution of Biofilm Producing Genes in Staphylococcus aureus Isolated from Local Cheese Samples in Maragheh City. Gene Cell Tissue, 4 (4) https://doi.org/10. 58 12/gct.66970

Feneley, R. C. L., Hopley, I. B. \& Wells, P. N. T. (2015). Urinary catheters: History, current status, adverse events and research agenda. Journal of Medical Engineering and Technology, 39, 459-470. https://doi.org/10. 31 09/ 0309 1902.2015.1085600

Herath, H. M. L. I., Senanayeke, D. M. N., Seneviratne, G. \& Bandara, D. C. (2013). Variation of Biochemical Expressions of Developed Fungal-Bacterial Biofilms over their Monocultures and its Effect on Plant Growth. Journal of tropical agricultural research, 24 (2), 186-192. http:// 19 2.248.4 3.136/handle/1/2279

Janda, J. M. \& Abbott, S. L. (2007). 16S rRNA gene sequencing for bacterial identification in the diagnostic laboratory: Pluses, perils, and pitfalls. Journal of Clinical Microbiology, 45 (9), 
2761-2764. https://doi.or g/10.1128/JCM.01228-07

Jenkins, C., Ling, C. L., Ciesielczuk, H. L., Lockwood, J., Hopkins, S., McHugh, T. D. \& Kibbler, C. C. (2012). Detection and identification of bacteria in clinical samples by $16 \mathrm{~S}$ rRNA gene sequencing: Comparison of two different approaches in clinical practice. Journal of Medical Microbiology, 61 (4), 483-488. https://doi.org/10.1099/j mm.0.030387-0

Kai, S., Matsuo, Y., Nakagawa, S., Kryukov, K., Matsukawa, S., Tanaka, H. \& Hirota, K. (2018). Direct PCR amplification of 16S rRNA genes offers accelerated bacterial identification using the MinIONTM nanopore sequencer. Biorxiv.Org. https: / / doi.org/10.1101/435859

Kırmusaoğlu, S. (2019). The Methods for Detection of Biofilm and Screening Antibiofilm Activity of Agents. Antimicrobials, Antibiotic Resistance, Antibiofilm Strategies and Activity Methods. https://doi.org/ 1 0.5772/intechopen.84411

Kotaskova, I., Obrucova, H., Malisova, B., Videnska, P., Zwinsova, B., Peroutkova, T. \& Freiberger, T. (2019). Molecular techniques complement culture-based assessment of bacteria composition in mixed biofilms of urinary tract catheter-related samples. Frontiers in Microbiology, 10. https://doi.org/10.3389/fmicb.2019.00462

Lu, L., Hu, W., Tian, Z., Yuan, D., Yi, G., Zhou, Y. \& Li, M. (2019). Developing natural products as potential anti-biofilm agents. Chinese Medicine (United Kingdom), 14 (1), 1-17. https://doi.org/10.1186/s13020-019-0232-2

Maciel, B. M., Santos, A. C. F., Dias, J. C. T., Vidal, R. O., Dias, R. J. C., Gross, E. \& Rezende, R. P. (2009). Simple DNA extraction protocol for a $16 \mathrm{~S}$ rDNA study of bacterial diversity in tropical landfarm soil used for bioremediation of oil waste. Genetics and Molecular Research, 8 (1), 375388. https://doi.org/10.4238/vol8-1gmr559

Magana, M., Sereti, C., Ioannidis, A., Mitchell, C. A., Ball, A. R., Magiorkinis, E. \& Tegos, G. P. (2018). Options and 
limitations in clinical investigation of bacterial biofilms. Clinical Microbiology Reviews, $31 \quad$ (3), 1-49. https: / / doi.org/ 10.1128/CMR.00084-16

Maharjan, G., Khadka, P., Siddhi Shilpakar, G., Chapagain, G. \& Dhungana, G. R. (2018). Catheter-Associated Urinary Tract Infection and Obstinate Biofilm Producers. Canadian Journal of Infectious Diseases and Medical Microbiology, 2018. https://doi.org/10.1155/2018/7624857

Murugan, K., Selvanayaki, K. \& Al-Sohaibani, S. (2016). Urinary catheter indwelling clinical pathogen biofilm formation, exopolysaccharide characterization and their growth influencing parameters. Saudi Journal of Biological Sciences, 23 (1), 150-159. https://doi.org/10.1016/j.sjbs. 2015.04 .016

Murugan, S., Devi, P. U. \& John, P. N. (2011). Antimicrobial Susceptibility Pattern of Biofilm Producing Escherichia coli of Urinary Tract Infections. Current Research in Bacteriology, 4, 73-80. https://doi.org/10.3923/crb. 2011. 73.80

Okuda, K. I., Yoshii, Y., Yamada, S., Chiba, A., Hironaka, I., Hori, S. \& Mizunoe, Y. (2018). Detection of bacterial DNA from central venous catheter removed from patients by next generation sequencing: A preli minary clinical study. Annals of Clinical Microbiology and Antimicrobials, 17. https://doi.org/10.1186/s12941-018-0297-2

Osungunna, M. O. \& Onawunmi, G. O. (2018a). Antibiotic resistance profiles of biofilm-forming bacteria associated with urine and urinary catheters in a tertiary hospital in Ile-Ife, Nigeria. Southern African Journal of Infectious Diseases, 33 (3), 80-85. https://doi.org/10.1080/23120 053.2018.1442192

Patel, F., Goswami, P. \& Khara, R. (2016). Detection of Biofilm formation in device associated clinical bacterial isolates in cancer patients. Sri Lankan Journal of Infectious Diseases, 6 (1), 43. https://doi.org/10.4038/ sljid.v6i1.8086

Pérez-Osorio, A. C., Williamson, K. S. \& Franklin, M. J. (2010). 
Heterogeneous rpoS and rhlR mRNA levels and 16S rRNA/rDNA (rRNA Gene) ratios within Pseudomonas aeruginosa biofilms, sampled by laser capture microdissection. Journal of Bacteriology, 192 (12), 29913000. https://doi.org/10.1128/JB.01598-09

Petrachi, T., Resca, E., Piccinno, M. S., Biagi, F., Strusi, V., Dominici, M. \& Veronesi, E. (2017). An alternative approach to investigate biofilm in medical devices: A feasibility study. International Journal of Environmental Research and Public Health, 14. https://doi.org /10.3390/ ijerph 14121587

Roy, S. S., Dasgupta, R. \& Bagchi, A. (2014). A Review on Phylogenetic Analysis: A Journey through Modern Era. Computational Molecular Bioscience, 04 (03), 39-45. https: //doi.org/10.4236/cmb.2014.43005

Sabir, N., Ikram, A., Zaman, G., Satti, L., Gardezi, A., Ahmed, A. \& Ahmed, P. (2017). Bacterial biofilm-based catheterassociated urinary tract infections: Causative pathogens and antibiotic resistance. American Journal of Infection Control, 45, 1101-1105. http//doi.org/10.1016/j.ajic .2017 .05 .009

Saini, H., Vadekeetil, A., Chhibber, S. \& Harjai, K. (2017). Azithromycin-Ciprofloxacin-Impregnated Urinary Catheters Avert Bacterial Colonization, Biofilm Formation, and Inflammation in a Murine Model of Foreign-BodyAssociated Urinary Tract Infections Caused by Pseudomonas aeruginosa. Journal of American Society for Microbiology, 61 (3), 1-13. https://doi.org/ 10.1128 / AAC. 01906-16

Sayal, P., Singh, K. \& Devi, P. (2014). Detection of bacterial biofilms in patients with indwelling urinary catheter. CIBTech Journal of Microbiology, 3 (3), 9-16. http://www. cibtech.org/cjm.htm

Segev, G., Bankirer, T., Steinberg, D., Duvdevani, M., Shapur, N. K., Friedman, M. \& Lavy, E. (2013). Evaluation of Urinary 
Catheters Coated with Sustained-Release Varnish of Chlorhexidine in Mitigating Biofilm Formation on Urinary Catheters in Dogs. Journal of Veterinary Internal Medicine, 27 (1), 39-46. https://doi.org/10.1111/j.1939-1676. 2012. $01027 . \mathrm{x}$

Singha, P., Locklin, J. \& Handa, H. (2017). A review of the recent advances in antimicrobial coatings for urinary catheters. Acta Bio materialia, 50, 20-40. https:// doi.org / 10.1016 / j.actbio.2016.11.070

Stickler, D. J. (2014). Clinical complications of urinary catheters caused by crystalline biofilms: Something needs to be done. Journal of Internal Medicine, 276, 120-129. https: // doi . org/10.1111/joim.12220

Tiwari, A. A. \& Ghnawate, N. (2017). Detection of Biofilm Forming Bacterial Communities from Urinary Catheter of Patients with Change in Its Antibiotic Susceptibility Pattern and Triclosan Effect from Different Hospitals of Amravati City Maharashtra, India. Open Journal of Medical Microbiology, $O 7$ (03), 51-66. https://doi.org/10.4236/ojmm.2017.73005

Wang, L., Zhang, S., Keatch, R., Corner, G., Nabi, G., Murdoch, S. \& Zhao, Q. (2019). In-vitro antibacterial and antiencrustation performance of silver-polytetrafluoroethylene nanocomposite coated urinary catheters. Journal of Hospital Infection. https://doi.org/ 10.1016/ j.jhin. 2019. 02.012

Wijesinghe, G., Dilhari, A., Gayani, B., Kottegoda, N., Samaranayake, L. \& Weerasekera, M. (2019). Influence of Laboratory Culture Media on in vitro Growth, Adhesion, and Biofilm Formation of Pseudomonas aeruginosa and Staphylococcus aureus. Medical Principles and Practice, 28 (1), 28-35. https://doi.org/10.1159/000494757

Yang, B., Wang, Y. \& Qian, P. Y. (2016). Sensitivity and correlation of hypervariable regions in 16S rRNA genes in phylogenetic analysis. BMC Bioinformatics, 17 (1), 1-8. https: / / doi.org/ 10.1186/s12859-016-0992-y

Ziemert, N. \& Jensen, P. R. (2012). Phylogenetic approaches to 
The Role of Bacterial Biofilms in Infections and Effective Prevention Strategies for Biofilm Formation in Urinary Catheters

natural product structure prediction. Methods in Enzymology, 517, 161-182. https:// doi.org/ 10.1016 / B9 78-0-12-404634-4.00008-5 